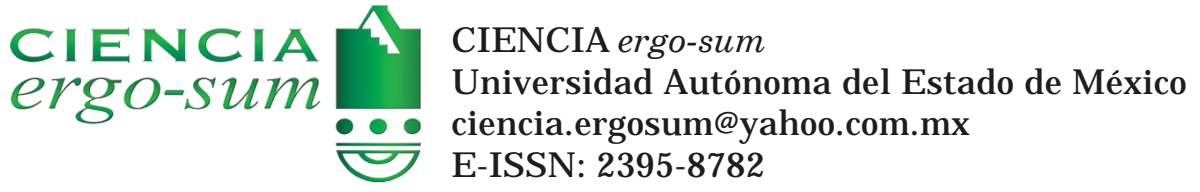

\title{
Instrumento de cobertura: Heston en MexDer
}

\author{
Najera López, M. de L.; Gutiérrez, R. deJ . \\ Instrumento de cobertura: Heston en MexDer \\ CIENCIA ergo-sum, vol. 28, núm. 3, noviembre 2021-febrero 2022 | e137 \\ Universidad Autónoma del Estado de México, México
}

Esta obra está bajo una Licencia Creative Commons Atribución-N oComercial-SinDerivar 4.0 Internacional .

Najera López, M. deL. y Gutiérrez, R. deJ . (2021). Instrumento de cobertura: Heston en MexDer. CIE NCIA ergo-sum, 28(3). https://doi.org/10.30878/ces.v28n3a9 


\title{
Instrumento de cobertura: Heston en MexDer Coverage instrument: Heston at MexDer
}

\author{
Ma. de Lourdes Najera López \\ Universidad Autónoma del Estado de México, México \\ mlnajeral@uaemex.mx \\ (1) http://orcid.org/0000-0002-6824-9561 \\ Raúl de Jesús Gutiérrez \\ Universidad Autónoma del Estado de México, México \\ rdejesusg738@profesor.uaemex.mx \\ (D) http://orcid.org/0000-0001-6878-3038
}

Recepción: 08 de octubre de 2020

Aprobación: 14 de junio de 2021

\section{Resumen}

Se valida el modelo de Heston (1993) para opciones sobre MXN/USD calculando la diferencia que existe entre la prima teórica y la que emite el Mercado Mexicano de Derivados (MexDer). Se estimaron los parámetros para el periodo 2003-2018 y se calcularon las primas de opciones europeas de compra (call) y venta (put) considerando los precios de ejercicio para el 9 de noviembre de 2018. Los resultados revelan sobrevaloración de opciones call OTM y ITM con una variación de 5.87\% y $3.46 \%$ respectivamente y para opciones put OTM con un $11.41 \%$, por lo que se ratifica que el modelo es confiable. Además se confirma que es un instrumento de cobertura para todos aquellos inversionistas expuestos al riesgo del mercado cambiario.

PaLABras ClaVe: precios de opción, tipo de cambio, volatilidad estocástica, estimación paramétrica.

\begin{abstract}
The Heston model (1993) is validated for MXN/USD options, calculating the difference between the theoretical premium and the one issued by the Mexican Derivatives Market. The parameters were estimated covering a period 2003-2018 and the prices of the European Call and Put options were calculated, considering the exercise prices of November 9, 2018. The results reveal overvaluation of Call OTM and ITM options with a variation of $5.87 \%$ and $3.46 \%$, respectively, and for Put OTM options with $11.41 \%$, thus ratifying that the model is reliable, it is also confirmed that it is a hedging instrument for all those investors exposed to the exchange market risk.
\end{abstract}

KEYWORDS: option prices, exchange rate, stochastic volatility, parametric estimation.

\section{INTRODUCCIÓN}

Los derivados financieros se han tornado relevantes debido a su influencia en el desempeño de diversos organismos de esa índole. La desregulación financiera ha establecido nuevas condiciones de competencia entre los intermediarios, lo que produjo una serie especulativa de burbujas de gran dimensión y de carácter irracional. Ante esto, surge la necesidad de protegerse de múltiples y crecientes riesgos en las operaciones, así como el sobrendeudamiento y quiebra de las corporaciones financieras y no financieras y a la vez de la persistente inestabilidad en las expectativas de rentabilidad de los activos financieros y no financieros (Schiller, 2003; Stiglitz, 2002).

Y es cuando la administración de riesgos, por medio del instrumento derivado opciones sobre divisas, es muy útil para la prevención del riesgo de mercado que enfrentan los inversionistas, empresarios y gobiernos.

En la literatura sobre cómo se han estimado los parámetros del modelo de Heston (1993) se han aplicado diferentes técnicas como los método generalizado de momentos (GMM) con su extensión al contexto de simulación (MMS), el método de máxima verosimilitud, los métodos de simulación Montecarlo y su extensión al marco bayesiano, así como los algoritmos de optimización no lineal tal como el de Nelder-Mead. 
En el contexto de los modelos de difusión, Hansen (1982) es el pionero en aplicar los modelos GMM para múltiples activos financieros. Para la valuación del precio de las opciones sobre divisas, Scott (1987) y Chesney y Scott (1989) encontraron que el GMM revela estimaciones sesgadas en los parámetros, lo que conlleva a una divergencia entre las primas teóricas y de mercado. De igual manera, Liu (1998) lo hace con los parámetros del modelo de Heston (1993) para tasas de interés mensuales, donde asume un valor inicial constante. Este supuesto incorrecto proporcionó estimaciones inconsistentes en los parámetros debido a la presencia de no estacionariedad en los precios de las acciones. Por su parte, Pan (1998) también modifica el GMM para incluir la volatilidad estocástica en la valuación de los precios de las opciones sobre divisas. Sin embargo, los hallazgos confirman precios teóricos sesgados debido a la inconsistencia de los parámetros.

Para relajar las debilidades del GMM, Bakshi et al. (1997) proponen una extensión al modelo de momentos generalizado e incorporan dos procesos markovianos estacionarios para la estimación de los modelos de Heston (1993) y Scott (1987). Los resultados revelan que el MMS[1] proporciona estimadores más eficientes, puesto que las primas presentan menor discrepancia respecto a las del mercado cuando el número de réplicas es suficientemente grande. De igual forma, los resultados Chernov y Ghysels (1998) reflejan este problema al usar la misma metodología.

Otro método que evalúa los parámetros es el del filtro de Kalman, que es subóptimo porque el problema de filtrar la volatilidad es sustancialmente no gaussiano y no lineal, y no es fácil de extender a datos de opciones, ya que estudios como el de Medina y Rodríguez (2010) lo usan en el caso de los parámetros de Heston (1993) para el índice de acciones que cotiza en la bolsa de Colombia de 2001 a 2005, y la experiencia muestra que implica mayor costo computacional y los resultados presentan sesgos por la volatilidad esperada sobre el resto del tiempo de vencimiento de la opción.

Por su parte, Andersen y Sørensen (2010) lo hacen de forma no paramétrica, esto es, evalúan conjuntamente los procesos de difusión contenidos en el modelo de Heston (1993) y fundamentan el efecto de apalancamiento, además de considerar la volatilidad con cambios bruscos (Mykland y Zhang, 2009).

El estudio de Singh y Dixit (2016) valora los parámetros de Heston (1993) por varios métodos y los compara por medio de datos del índice CNX Nifty del mercado de la India, entre los cuales se tiene la optimización heurística con dos variables de evolución diferencial, el algoritmo de Nelder-Mead, que no requiere derivar la función objetivo por el empleo de un algoritmo métrico variable. Asimismo, se valen del método cuasi-Newton que utiliza una aproximación del hessiano, del método del gradiente conjugado no lineal que se ocupa para encontrar el mínimo local de una función no lineal sólo con su gradiente, de otro método que encuentra el mínimo local de una función no lineal, discontinua y estocástica y que trabaja sin restricciones y también del método cuasi-Newton de comunicación inversa, que se apoya del enfoque de la región de confianza. Los resultados muestran que este último es estable y eficiente, por lo que otorga más precisión en los precios de las opciones que los demás.

Para este estudio, se empleará la metodología de Nelder-Mead por ser método simplex no lineal seguro para hallar óptimos de una función.

\section{Modelación matemática FinanCiera}

El modelo de Heston (1993) presenta un proceso de difusión para el precio de la opción, que es similar al de Black y Scholes (1973) con la diferencia de que la volatilidad conlleva un proceso estocástico representado por las ecuaciones diferenciales estocásticas de Ornstein-Unlenbeck:

$$
\begin{gathered}
d S_{t}=\mu S_{t} d t+\sqrt{\sigma_{t}} S_{t} d W_{s} \\
d \sigma_{t}=\kappa\left(\theta-\sigma_{t}\right) d t+\sigma \sqrt{\sigma_{t}} d W_{\sigma}
\end{gathered}
$$


Para considerar el efecto de apalancamiento los procesos de Wiener $W_{S}$ y $W_{\sigma}$ deben estar correlacionados $d W_{S}$ $d W_{\sigma}=\rho d t$, además de considerar $\theta$, que representa el valor promedio de la varianza en un largo plazo, $\kappa$ la velocidad de reversión al promedio y $S_{t}$ el precio del activo subyacente. También, contiene a la volatilidad $\sigma_{t}$ de la varianza $\sqrt{\sigma_{t}}$, que permite valorar los instrumentos derivados, es decir, adaptarse a la sensibilidad de dicha variabilidad estocástica, por ejemplo, si $\sigma=0$ (Heston, 1993), además $\mu$ es el parámetro de tendencia del activo subyacente.

El proceso de la evolución de la divisa bajo el promedio de probabilidad neutral al riesgo está definido por:

$$
\frac{d S_{t}}{S_{t}}=\mu d t+\sqrt{\sigma_{t}} d W_{S, t}
$$

En seguida se aplica el lema de Itô y se simplifica para obtener el proceso para la varianza $\sigma t$ :

$$
d \sigma_{t}=\kappa\left[\theta-\left(1+\frac{\lambda}{\kappa}\right) \sigma_{t}\right] d t+\sigma \sqrt{\sigma_{t}}\left[\frac{\lambda}{\sigma} \sqrt{\sigma_{t}} d t+d W_{\sigma, t}\right] d \sigma_{t}=\kappa^{*}\left(\theta^{*}-\sigma_{t}\right) d t+\sigma \sqrt{\sigma_{t}} d W \underset{\sigma, t}{Q}
$$

La varianza de largo plazo se representa por el parámetro $\theta^{*}$ y la tasa de reversión a la media por $\kappa^{*}$.

La prima de la opción europea de compra (call) sobre tipo de cambio, que considera el precio spot $S_{t}$ de la divisa y $K$ el precio de ejercicio de la opción, además del vencimiento $\tau=T-t$, es:

$$
C=e^{-r \tau} \int_{K}^{\infty}\left(S_{T}-K\right) f\left(S_{\tau}\right) d S_{\tau}
$$

Si se consideran las ecuaciones (4) y (5) y se utiliza la transformada inversa de Fourier como lo hace Heston (1993), condicionada a la varianza de los rendimientos de los precios del activo subyacente, la opción call europea sobre divisa está dada por:

$$
C=S e^{-r} f^{\tau} P_{1}-K e^{-r} d \tau P_{2}
$$

donde

$$
P_{j}=\frac{1}{2}+\frac{1}{\pi} \int_{0}^{\infty} \operatorname{Re}\left[\frac{e^{-i z \ln (K) f_{j}}}{i z}\right] d z
$$

con $i=\sqrt{-1}$ y $f_{j}$ para $j=1,2$, de la siguiente manera:

$$
\begin{gathered}
f_{j}=e C_{j}+D_{j \sigma_{0}}+i z \ln \left(S_{0}\right) \\
C_{j}=r i z T+\frac{\kappa \theta}{\sigma^{2}}\left[\left(b_{j}-p \sigma i z+d_{j}\right) T-2 \ln \left(\frac{1-g_{j} e^{T d j}}{1-g_{j}}\right)\right] \\
g_{j}=\frac{b_{j}-p \sigma i z+d_{j}}{b_{j}-p \sigma i z-d_{j}} \\
d j=\left[\left(\rho \sigma i z-b_{j}\right)^{2}-\sigma^{2}\left(2 u_{j} i z-z^{2}\right)\right]^{\frac{1}{2}}
\end{gathered}
$$

En seguida, se efectúan la integral de la ecuación (7), y al considerar la expresión (6), se obtiene la prima (C) de una opción de compra.

Por otro lado, al considerar la inexistencia de arbitraje para el precio del call (C) y la paridad put-call se obtiene el precio para la opción put $(\mathrm{P})$ :

$$
P=C-S e^{-r} f^{\tau}+K e^{-r} d^{\tau}
$$




\section{Aplicación al mercado meXicano}

Para la estimación de los parámetros y su validación se consideran los precios diarios del tipo de cambio MXN/ USD obtenidos de la base DataStream con el periodo comprendido del 14 de abril de 2003 al 30 de noviembre de 2018, donde se contaron 3830 datos. El periodo de la muestra está determinado con el fin de considerar el inicio de las operaciones del instrumento derivado "opciones sobre divisa" en el Mercado Mexicano de Derivados (MexDer), así como la crisis financiera de 2009 y las turbulencias de los mercados hasta 2018.

Dada la prima disponible de la opción call, se estimaron los parámetros de Heston (1993) de tal manera que la prima de la opción obtenida esté muy cerca de los precios del mercado. Para ello, primero se evalúan los parámetros por medio de Nelder-Mead teniendo en cuenta un vector inicial descrito por los estadísticos descriptivos y finalmente comparar las variaciones de los precios teóricos respecto a los de mercado.

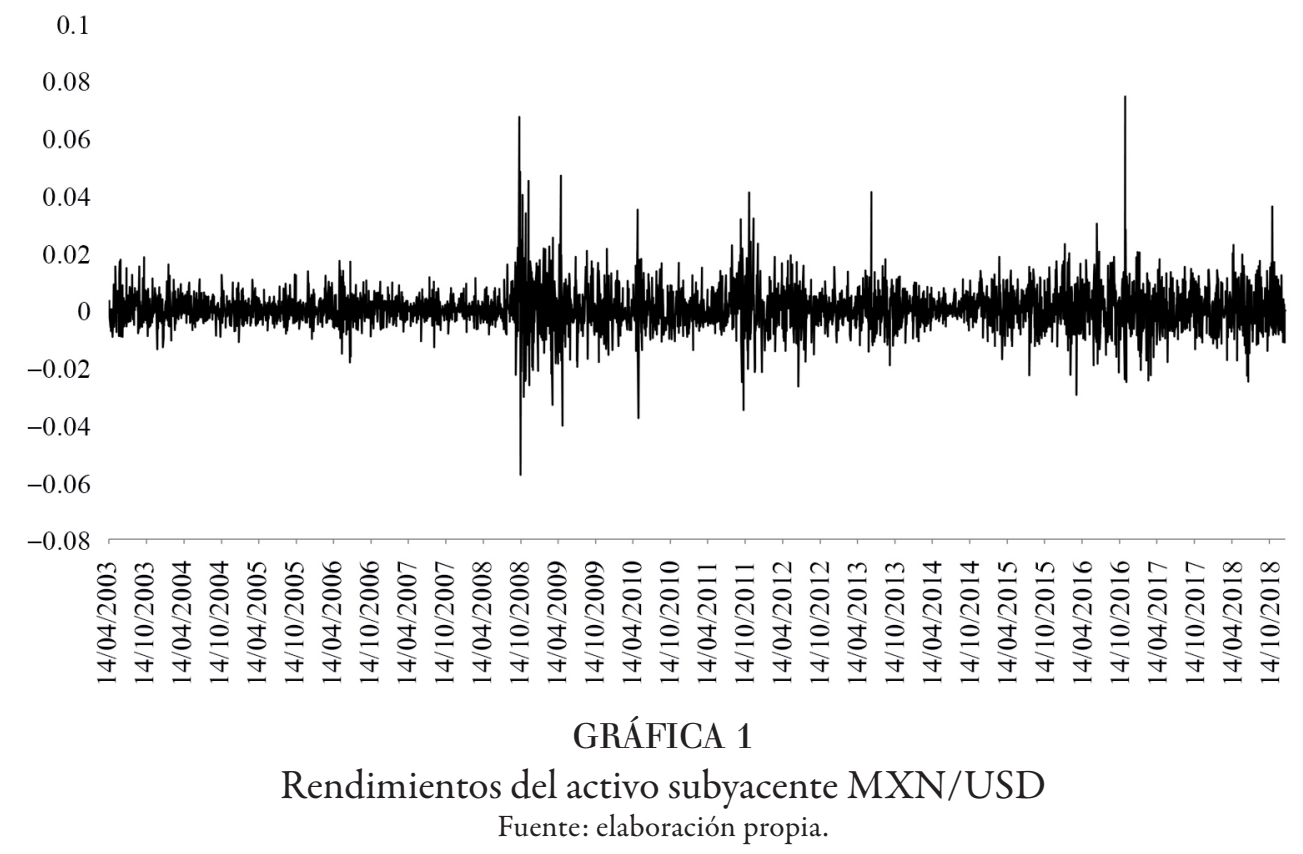

Se aprecia que el tipo de cambio experimenta gran volatilidad (gráfica 1), cambios bruscos e inesperados, sobre todo durante la crisis financiera global en 2008, en 2011 con los desastres naturales de Asia y la crisis en la zona euro y en 2012 surge la incertidumbre generada sobre la magnitud del ajuste fiscal previsto para 2013 en Estados Unidos. Por todo lo anterior, cabe resaltar la importancia de llevar a cabo modelos que consideren la variabilidad estocástica del activo subyacente en el cálculo de la prima de la opción para que los agentes económicos, financieros y administradores de riesgos se protejan de las eventualidades que aparecen en los mercados financieros.

Empleando la metodología descrita, se obtiene el resumen de estadísticos descriptivos (cuadro 1) para establecer el vector inicial, alimento del algoritmo que dará los parámetros estimados. Además, se llevaron a cabo las pruebas de heteroscedasticidad para comprobar que los rendimientos presentan volatilidad estocástica (Lin et al., 2001; Azencott et al., 2015; Mondal et al., 2017) y es estacionaria para obtener precios más justos.

Los resultados del cuadro 1 indican que la serie de rendimientos de la divisa es sesgada positiva y leptocúrtica, es decir, muestra propiedades de colas más anchas, particularmente la cola derecha. La asimetría positiva señala que los rendimientos positivos extremos (una depreciación de la moneda mexicana) tienen mayor probabilidad de ocurrir. Por su parte, el $p$-valor del estadístico de Jarque-Bera indica que no existe normalidad en los rendimientos del activo subyacente a un nivel de significancia de $5 \%$ y las pruebas de raíces unitarias revelan que la serie es estacionaria; de acuerdo con lo anterior, se comprueba que presentan volatilidad estocástica (gráfica 1). 


\section{CUADRO 1}

Estadísticos descriptivos y pruebas

\begin{tabular}{|c|c|c|}
\hline 2003-2018 & & Diarios \\
\hline Variables & & Rendimientos MXN/USD \\
\hline \multirow{10}{*}{ 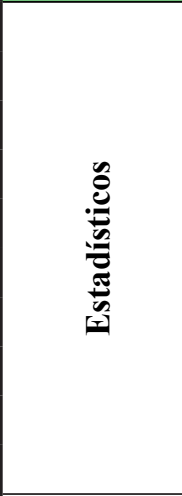 } & Media & 0.0159 \\
\hline & Mediana & -0.0195 \\
\hline & Desviación estándar & 0.7162 \\
\hline & Varianza & 0.5130 \\
\hline & Asimetría & 0.6836 \\
\hline & Curtosis & 12.6188 \\
\hline & Máximo & 7.3724 \\
\hline & Mínimo & -5.7561 \\
\hline & Jarque-Bera & 15196.8 \\
\hline & $p$-valor & 0.0000 \\
\hline \multirow{4}{*}{ 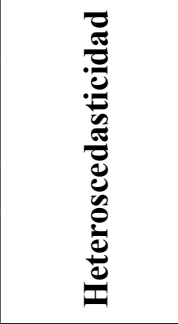 } & \multicolumn{2}{|c|}{ Prueba de White } \\
\hline & $F$-estadístico & 53.4875 \\
\hline & No. Obs* $\mathrm{R}^{2}$ & 52.7841 \\
\hline & $p$-valor & 0.0000 \\
\hline \multicolumn{3}{|c|}{ Prueba de raíces unitarias } \\
\hline \multirow{4}{*}{ ADF } & $t$-estadístico & -60.667 \\
\hline & $p$-valor & 0.0000 \\
\hline & Durbin-Watson & 1.9992 \\
\hline & $t$-estadístico & -60.650 \\
\hline \multirow[t]{2}{*}{ PP } & $p$-valor & 0.0000 \\
\hline & Durbin-Watson & 1.9992 \\
\hline
\end{tabular}

Fuente: elaboración propia.

A continuación, en el cuadro 2 se estiman los parámetros de Heston (1993).

\section{CUADRO 2}

Parámetros estimados de Heston (1993)

\begin{tabular}{|c|c|c|c|c|}
\hline \multicolumn{5}{|c|}{ Periodo 14/04/2003-09/11/2018 } \\
\hline Periodo & Diarios & Semanales & Quincenales & Mensuales \\
\hline$\mu$ & 0.0596 & 0.2528 & 0.7326 & 2.3458 \\
\hline$\theta$ & 0.0185 & 0.0851 & 0.2055 & 0.7289 \\
\hline$\sigma$ & 0.2996 & 0.0479 & 0.0866 & 0.1651 \\
\hline$\kappa$ & 4.9002 & 5.9948 & 8.0147 & 13.3759 \\
\hline$\rho$ & 0.0014 & 0.1836 & 0.5241 & 0.6822 \\
\hline fval & 1 & 1 & 1 & 1 \\
\hline exitflag & 1 & 1 & 1 & 1 \\
\hline iteraciones & 13 & 13 & 13 & 14 \\
\hline funcCount & 90 & 90 & 90 & 97 \\
\hline$h i, p$-valor & $0,0.789$ & $0,0.653$ & $0,0.682$ & $0,0.489$ \\
\hline
\end{tabular}

Fuente: elaboración propia. 
Los resultados expuestos (gráfica 1) muestran parámetros consistentes a través de la prueba de Kolmogorov-Smirnov (doble muestra), donde señala que valores reales y los simulados provienen de la misma distribución al presentar un $p$-valor mayor a 0.05 .

Posteriormente, se determinan las primas de las opciones call y put por medio del modelo de Heston (1993), las cuales se comparan con las del mercado que cotizan en el MexDer. Las primas corresponden a 57 precios de ejercicio (con incrementos de 5 centavos) cuando la opción se encuentra fuera del mercado (OTM), 63 dentro del mercado (ITM) y solamente 1 para cuando el precio de ejercicio es igual al precio del activo subyacente (ATM).
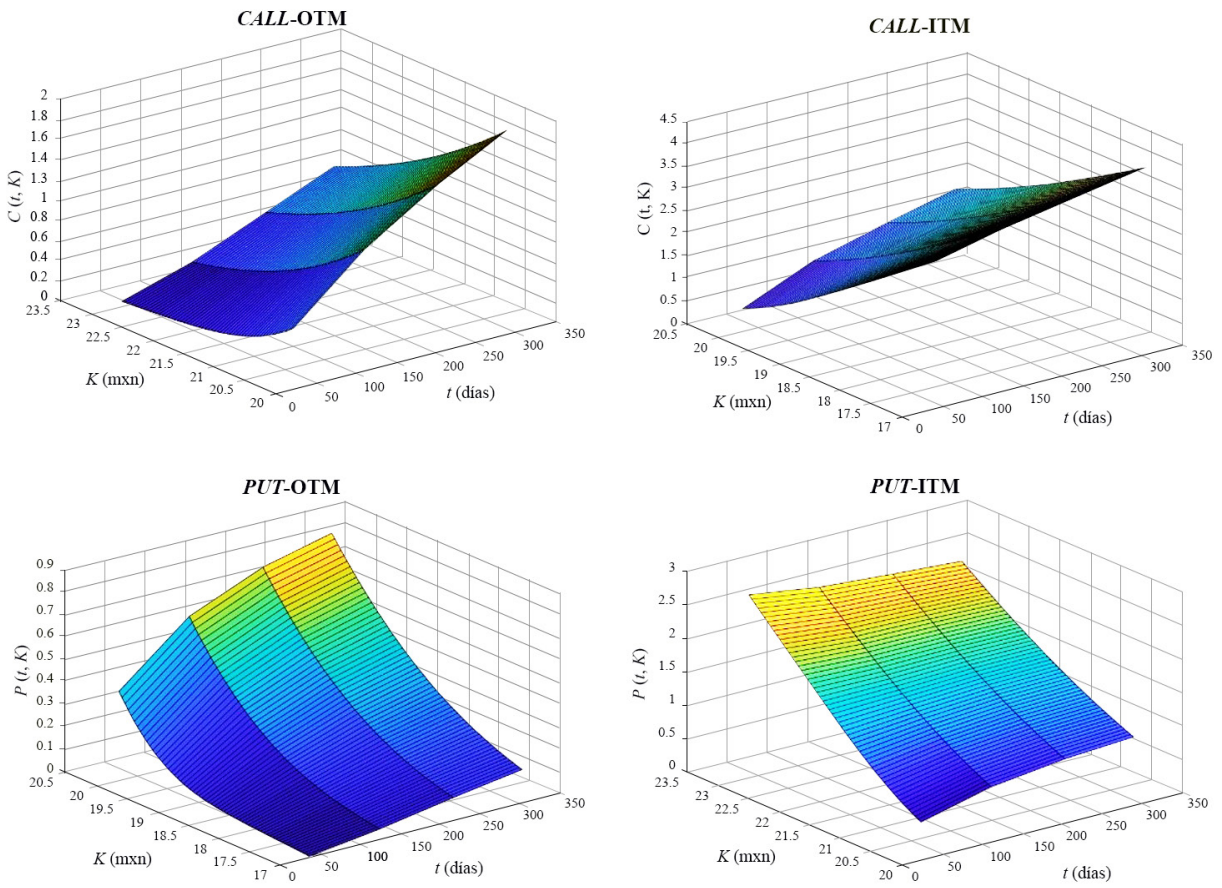

GRÁFICA 2

Precio de la opción

Fuente: elaboración propia.

El estudio muestra que el modelo de Heston (1993) sobrevalora la prima de las opciones call OTM e ITM (gráfica $2 \mathrm{a} \mathrm{y} 2 \mathrm{~b}$ ) con una diferencia mínima (5.87\%) en comparación con los precios del mercado para tiempos próximos por vencer ( 38 días) con precios de ejercicio muy profundamente fuera del mercado y con una variación en ITM de 3.46\%. También refleja en opciones call que cuando mayor es el tiempo por vencer y con precios de ejercicio cercanos a los del mercado la prima de la opción presenta la máxima variación de los precios en un $21.51 \%$.

Para las opciones put el precio es sobrevalorado y mucho más justo cuando están dentro del mercado, por lo que queda evidenciada la mayor prima para opciones muy profundamente ITM próximas a vencer, equivalente a $13.88 \%$ (gráfica 2 c y $2 \mathrm{~d}$ ) y un mínimo para precios de ejercicio cercanos a los precios del mercado.

Lo anterior verifica que el modelo de Heston (1993) es confiable para calcular los precios de las opciones sobre moneda al considerar la volatilidad estocástica y también otorga soluciones semianalíticas para los precios de las opciones europeas, por lo cual es más fácil estimar sus parámetros.

Es importante resaltar que Heston (1993) en su estudio teórico sobrevalora las opciones OTM, lo cual coincide con los resultados del presente trabajo para la divisa MXN/USD. De igual manera, Bakshi et al. (1997), Bates (1996) y Abraham y Taylor (1993) replican dicho modelo y encontraron que otorga precios 
más justos a los del mercado. Asimismo, Levendorskiu (2012) aplica el modelo de Heston (1993) para la fijación de precios sobre las opciones europeas del índice Euro-Stoxx 50 y la divisa AUD/JPY y Crisóstomo (2014) lo hace para opciones call europeas sobre el Nasdaq que al valuar el precio de la opción muestra resultados robustos. También, Azencott et al. (2015) avalan la efectividad del modelo de Heston (1993), el cual replican para el índice S\&P 500; este estudio cuantifica el impacto de los errores de las opciones call europeas respecto a los precios reales, los cuales avalan que es un modelo eficiente. Para economías emergentes se tiene el estudio de Singh y Dixit (2016) que calibra el modelo de Heston (1993) para el índice CNX Nifty del mercado de la India, quienes encuentran que el modelo de volatilidad estocástica otorga resultados superiores a los de Black-Scholes. Además, Mondal et al. (2017) replican a Heston (1993) y descubren que la correlación entre los rendimientos del activo subyacente y la volatilidad estocástica capturan el efecto de apalancamiento que provoca efectos importantes en la prima de la opción. Roldán (2018) evalúa el modelo de Heston y Black-Scholes para el tipo de cambio colombiano/dólar estadounidense, el cual sustenta resultados semejantes al análisis de este artículo.

\section{Discusión}

Es importante señalar que las opciones financieras son un instrumento de cobertura para los inversionistas y no inversionistas, quienes transfieren el riesgo de pérdida en épocas de fuerte inestabilidad de los precios. Así, los compradores de opciones call y put cuando se valen de este instrumento presentan pérdidas limitadas ante volatilidades en el mercado cambiario, donde el costo de la prima de la opción es la máxima pérdida y no el valor total del activo como sucede con otros instrumentos.

Aunque el modelo de Heston (1993) es de cobertura, da la posibilidad de obtener beneficios ilimitados si se tiene una opción call cuando el precio del activo subyacente es alcista y si es de manera contraria sólo pierde la prima de la opción.

Finalmente, estimar los parámetros de Heston (1993) otorga resultados consistentes y contribuye a que los precios teóricos de las opciones (MXN/USD) estén más cerca de los del mercado que se negocia en el MexDer; a su vez, dicho modelo puede ser un instrumento de cobertura ante la volatilidad del mercado cambiario.

\section{Prospectiva}

En definitiva, contar con instrumento de cobertura que considera más factores macroeconómicos, como la volatilidad del activo subyacente y las tasas de interés domésticas y extranjeras, cuando presentan un comportamiento estocástico, garantiza reducciones de la exposición al riesgo de mercado cambiario para todos los agentes financieros y no financieros. En este sentido, para las empresas importadoras y exportadoras que confían sus flujos financieros pueden asegurar sus portafolios de inversión por medio de opciones. Los bancos centrales al usar dicho instrumento fortalecen la economía a través de una política monetaria más eficiente estabilizando el tipo de cambio y las tasas de interés y también crean un país más competitivo y confiable para la inversión extranjera e interna; aunado a ello, los bancos protegidos por dicho instrumento hará que sean más rentables y con mayor calificación crediticia.

Por lo anterior, el desarrollo de un modelo de tres factores (Heston-Hull-White) (Heston, 1993; Hull y White, 1987), con las características mencionadas, es un trabajo a futuro, además se pretende aplicar al MexDer y comprobar que se obtienen diferencias mínimas entre los precios teóricos y los del mercado. Cabe señalar que con Heston-Hull-White al valuar el precio de la opción sobre tipo de cambio se enfrenta a tres ecuaciones diferenciales estocásticas con siete parámetros por estimar, lo que implica la elaboración del algoritmo numérico lo más complejo que permita. 


\section{REFERENCIAS}

Abraham, A., \& Taylor, W.M. (1993). Pricing currency options with scheduled and unscheduled announcement effects on volatility. Managerial and Decision Economics, 14(4), 311-326.

Andersen, T. G., \& Sørensen, B. (2010). GMM estimation of a stochastic volatility model: A Monte Carlo study. Journal of Business and Economic Statistics, 14, 328-352.

Azencott, R., Gadhyan, Y., \& Glowinski, R. (2015). Option pricing accuracy for estimated Heston models. Quantitative Finance, 1-18.

Bates, D. (1996). Jumps and stochastic volatility: Exchange rate processes implicit in deutsche market options. The Reviews of Financial Studies, 9, 69-107.

Bakshi, C., Cao, C., \& Chen, Z. (1997). Empirical performance of alternative option pricing models. The Journal of Finance, 52(5), 2003-2049.

Black, F., \& Scholes, M. (1973). The pricing of options and corporate liabilities. Journal of Political Economy, 81(3), 637-654.

Chernov, M., \& Ghysels, E. (1998). A study towards a unified approach to the joint estimation of objective and risk neutral measures for the purpose of options valuation. Journal of Financial Economics, 56(3), 407-458.

Chesney, M., \& Scott, L. (1989). Pricing european currency options: A comparison of the modified blanck-scholes model and a random variance model. Journal of Financial and Quantitative Analysis, 24(3), 267-284.

Crisóstomo, R. (2014). An analysis of the Heston stochastic volatility model: Implementation and calibration using Matlab. CNMV Working Paper, 58.

Duffie, D., \& Singleton, K. (1993). Simulated moments estimation of Markov models of asset prices. Econometrica, 61(4), 929-952.

Hansen, L. P. (1982). Large sample properties of generalized method of moments estimators. Econometrica, 50(4), 1029-1054.

Heston, S. L. (1993). A closed-form solution for options with stochastic volatility with applications to bond and currency options. Review of Financial Studies, 6(2), 327-343.

Hull, J., \& White, A. (1987). The pricing of options on assets with stochastic volatilities. Journal of Finance, 42(2), 281-300.

Levendorskiĭ, S. (2012). Efficient pricing and reliable calibration in the Heston model. International Journal of Theoretical and Applied Finance, 15(7), 1250050.

Lin, Y. N., Strong, N., \& Xu, X. (2001). Pricing FTSE 100 index options under stochastic volatility. The Journal of Futures Markets, 21(3), 197-211.

Liu, J. (1998). Portfolio selection in stochastic environments. The Review of Financial Studies, 20(1), 1-39.

Medina, R. T. y Rodríguez, H. Y. (2010). Una revisión de los modelos de volatilidad estocástica. Comunicaciones en Estadistica, 3(1), 79-98. https://doi.org/10.15332/s2027-3355.2010.0001.05

Mondal, M. K., Alim, A., Rahman, F., \& Biswas, H. A. (2017). Mathematical analysis of financial model on market price with stochastic volatility. Journal of Mathematical Finance, 7(2), 351-365.

Mykland, P. A., \& Zhang, L. (2009). The Econometrics of High Frequency Data. In M. Kessler, A. Lindner, and M. Sorensen (Ed.), Statistical Methods for Stochastic Differential Equations (pp. 109-190). New York: Chapman and Hall/CRC.

Pan, J. (1998). Integrated time-series analysis of spot and option prices. AFA 2001 New Orleans Meetings. https://doi.org/10.2139/ssrn.196808 
Roldán, M. L. (2018). Pronóstico de divisas latinoamericanas con modelos de volatilidad estática y estocástica. Ingenieria, 23(2), 166-189.

Schiller, R. (2003, March 22). Risk management for the masses. The Economist.

Scott. L. O. (1987). Option pricing when the variance changes randomly: Theory, estimation, and application. Journal of Financial and Quantitative Analysis, 22, 419-438.

Singh, S., \& Dixit, A. (2016). Performance of the Heston's stochastic volatility model: A study in indian index options market. Theoretical Economics Letters, 6(2), 151-165.

Stiglitz, J. (2002). What I learned at the world economic crisis. The Insider. The New Republic. Robarts Centre for Canadian Studies.

\section{Notas}

[1] Para una explicación más detallada del método de momentos simulados consúltese a Duffie y Singleton (1993).

\section{BY-NC-ND}

